\title{
Mix and Match: Promoting Interdisciplinary Teaching, Learning, and Community through Classroom-level Partnerships
}

\author{
Joanne A. Fox, Natalie Baloy, and Allen Sens, University of British Columbia
}

\begin{abstract}
Developed at the University of British Columbia (UBC), UBC Mix creates interdisciplinary learning experiences for students by developing partnerships between existing courses, and between existing courses and other learning opportunities. UBC Mix connects faculty members interested in making their courses more interdisciplinary, and facilitates the creation of shared learning activities that "mix" students together. The UBC Mix project provides a model for facilitating interdisciplinary teaching and learning within existing courses and programs at colleges and universities.
\end{abstract}

\section{Introduction}

The benefits of interdisciplinary teaching and learning are well established (cf. Letterman and Dugan 2004; Knotts, Henderson, Davidson, and Swain, 2009; Smith and McCann 2001). Institutions of higher learning have responded by creating interdisciplinary degree programs, colleges, institutes and centres (cf. Holley et al. 2009; Klein 2009; Newswander and Borrego 2009). Individual instructors have also created courses with explicit interdisciplinary content and pedagogy (cf. Epstein 2004; Miller and Totten 2009; Newell 1994). UBC's strategic plan, Place and Promise (UBC 2012), speaks to the importance of learning across disciplines: 'Through rigorous study within and across disciplines, students acquire the knowledge, inquiry and communication skills, professional abilities, and understanding of other cultures that enhance their personal development and enable them to contribute and lead in a global society.' Like most universities, UBC operates a wide variety of excellent interdisciplinary programs. However, these programs require a significant commitment of resources, and do not serve the majority of students. In addition, individual instructors often introduce interdisciplinary themes into their courses, but may be constrained by time and expertise limitations, and a lack of awareness of related teaching elsewhere in the institution. In this article, we discuss the creation and conceptual design of the UBC Mix project as a model of interdisciplinary engagement. We critically reflect on the challenges and benefits of the Mix experience, and highlight how other educators and programs can adopt this approach to promote interdisciplinarity at their institutions.

\section{How the UBC Mix Project Started}

The UBC Mix Project developed under the auspices of the UBC Terry Project, a program dedicated to advancing interdisciplinary education across the arts and sciences. The Terry Project manages a number of learning activities, including the annual TEDx Terry Talks Conference featuring UBC students. A 2008 TEDx presentation by Geoff Costeloe, an Integrated Sciences and Political Science double major, was the genesis of the UBC Mix project. Geoff suggested developing a strategy to bring students in different disciplines together to discuss common 
themes and problems. The Terry Project Team adopted Geoff's idea and successfully applied to launch UBC Mix through the UBC Teaching and Learning Enhancement Fund (TLEF), which provides seed funding for new educational initiatives. In Geoff's own words, 'My Terrytalk was about the importance of integration in university education and integrative solutions in society. With UBC Mix, I hope to create more opportunities for students to experience issues through different disciplines and work with students from different backgrounds.'

\section{The UBC Mix Concept and Design}

UBC Mix was based on the value of learning across the disciplines, and the need to develop interdisciplinary instruction in ways that are accessible and achievable for most instructors. The objective of UBC Mix was to offer a mechanism for expanding and enhancing interdisciplinary opportunities for students, in a manner that is complementary with existing courses and programs. Emphasizing classroom-level learning, Mix offered a flexible and manageable solution for instructors committed to interdisciplinary instruction. The UBC Mix Project operated between 2009 and 2013.

During the academic year, Mix conducted two or three regularly scheduled workshops, or "Mixers" for faculty and staff. Workshop participants shared their Mix experiences, explored the possibility of new partnerships, discussed best practices and lessons learned, and met instructors and/or program staff interested in a potential partnership. The UBC Mix project was supported by a "storefront" webpage introducing Mix to the UBC community, and a Wiki Community Portal, which served as an online space to share resources and ideas, faculty contacts, and instructional resources. The process of facilitating collaboration between participants developed into an interdisciplinary community of practice that continues to serve as a focal point for sharing experiences, a repository of examples and expertise, and a space for face-to-face discussions of potential collaborations. Instructional resources and other Mix materials are now available on the Interdisciplinary Community of Practice website (blogs.ubc.ca/ubcmix).

The Mix project team was composed of two UBC faculty members and a coordinator. The faculty members provided the academic and institutional leadership for the UBC Mix project on a volunteer basis. The UBC Mix Coordinator was a part time position held by a graduate student. The Coordinator was responsible for identifying potential UBC Mix partners, connecting potential partners, engaging with educational support units on campus, developing promotional materials, maintaining the web presence, compiling and communicating Mix activity results and assessments, and facilitating Mix workshops. A core task was facilitating the logistics involved in joint class activities: booking larger classroom space, identifying a meeting time, and securing appropriate equipment.

To create new "Mixes" and support existing ones, the project actively recruited and engaged instructors. This was achieved through presentations at faculty meetings, contacting potential participants based on their teaching interests, communication with teaching and learning communities of practice, and advertisements at UBC events. Once a partnership was created, the UBC Mix coordinator and/or the faculty leads communicated with both partners to provide advice on the design of a Mix idea. If necessary, the UBC Mix Coordinator searched for a suitable partner on behalf of a faculty member. If potential "Mixes" required financial support, Mix participants submitted an application for funding. Through the UBC TLEF grant, the UBC Mix project provided seed funding to support the development and implementation of Mix activities ( $\sim 500$ per UBC Mix pairing), and offered support to help document and archive Mix activities, developed evaluation strategies, and provided standardized assessment mechanisms. 
Proposals were accepted twice a year and vetted by the Mix team. After the planned learning activities had taken place, participants submitted student feedback forms, which the coordinator compiled into brief assessment reports for faculty use. Faculty feedback was also compiled, and used in subsequent years to improve Mix events, procedures, and the quality of experience-based advice to Mix instructors.

\section{Examples of UBC “Mixes”}

By making small adjustments to "mix" their students together to explore a common theme, concept, or problem, partnered instructors created activities that enhanced learning across disciplines. Examples of such activities include common lectures, joint group projects, electronic discussions between classes, and field trips. These activities enhanced interdisciplinary communication, exposed students to new ideas, extended learning beyond a single classroom, and engaged students with communities both within and outside the University. At UBC, over twenty different UBC Mix activities reached more than 2400 students and involved partnerships between sixty courses and/or programs. Examples of past "Mixes" include:

\section{Shared Assignments}

Joint group research project: In a partnership between a first year History course and a Science One Biology course, instructors developed a joint assignment on native trees around campus, undertaking joint lectures, employing a shared course reading, and conducting joint film viewings.

Data mash-ups: Students in an advanced statistics course paired up with students in Biology and the School of Population and Public Health (SPPH) to prepare statistical reports for shared term projects. The students from biology and SPPH supplied fresh data for statistics students to use in as they explored different statistical models. Students collaborated to identify statistical needs and develop appropriate statistical analyses that were mutually beneficial to both partners.

\section{Common Experiences}

Reading, debating, sharing: UBC Reads Sustainability, a sustainability initiative on campus, invited authors of popular books to spark interdisciplinary discussion on global environmental issues. Mix promoted the events, and encouraged instructors to incorporate the books and author visits into their courses. Courses from Anthropology, Political Science, Biology, and Engineering, among others, participated in this Mix over its three-year partnership.

Film screening: An engineering course on environmental engineering and sustainability invited students enrolled in courses on film studies, the Arctic, snow and ice geology, and sustainability to watch Chasing Ice, an award-winning film about the changing Arctic landscape. After the screening, students engaged in discussion about the film from their various disciplinary perspectives.

Networking 
First years fraternizing with Frankenstein: With assistance from Mix, students in two first year interdisciplinary programs, Arts One and Science One, began an annual tradition: joining together to socialize and learn from one another. One year they planned a joint dinner and lecture. Most recently, they watched the film Frankenstein and discussed its moral and scientific implications.

Communicating beyond the university: TerreWeb, an interdisciplinary project designed to promote science communication, hosted two networking fieldtrips to meet partner institutions, offered innovative social media and film production workshops, and scheduled a series of lectures on public science issues.

\section{Community Engagement}

Learning from the experience of homelessness: In partnership with the UBC Community Learning Initiative, this multi-year Mix explored themes of poverty, inequality, and development by hosting an education specialist on homelessness and an Aboriginal man who has spent many years on the streets of Vancouver's Downtown Eastside. Students from Economics, Sociology, Education, and Applied Science watched a short film about his life and a lecture on homelessness before engaging in discussion.

International service learning: Senior Philosophy and Sociology students travelled to Guatemala through UBC's Go Global service learning program, participating in guest lectures by local community members and sharing readings and fieldtrips to a number of sites.

\section{Impact and Assessment}

Among several project evaluation measures, the key indicator of UBC Mix success has been positive student feedback and evidence of achieved learning objectives. Mix developed a standardized assessment mechanism for all UBC Mix partnerships. The Mix Coordinator collected, analyzed, and documented feedback specific to individual UBC Mix activities and developed Mix feedback reports. These reports were shared with instructors and other members of the Mix community to enhance interdisciplinary teaching practice and stimulate future Mix ideas.

On Mix feedback forms, students defined interdisciplinarity and described its values from their perspectives. They emphasized that interdisciplinary learning offers new and different perspectives, opens opportunities for knowledge exchange and collaborative learning, and creates moments for 'real' world application, One student commented, 'Interdisciplinary learning is a fantastic idea to expand and share knowledge across faculties that tend to be quite closed and separate.' The majority of student respondents commented favorably on their participation in common experiences facilitated by UBC Mix. They expressed appreciation for the opportunity to engage with students from other disciplines and emphasized the importance of interdisciplinary collaboration for expanding perspectives, problem-solving, and developing innovative ideas and approaches to familiar topics. According to one student, UBC Mix offers ' $a$ good way of meeting students who come from different learning backgrounds... this is more like what we would experience in the real world.'

\section{Challenges and Responses}


The UBC Mix project encountered a number of challenges as it evolved, and through this experience valuable lessons were learned which informed our responses.

\section{Instructor Time Constraints}

Even the most dedicated instructors have practical limitations on their time and their availability for workshops, forging interdisciplinary partnerships, and changing their existing courses. The Mix project recognized this constraint by providing valuable facilitation services through the Mix Coordinator, who was available in person and by email, and by creating an online repository of examples and resources for participants. Mix workshops were held 2-3 times per year and resources featured short takeaway pieces that could be readily applied by instructors.

\section{Course Timetables and Space}

Holding common classes or other Mix activities was easier if partnered courses were scheduled on the same day and at the same time. While not insurmountable, scheduling problems did have a significant impact on Mix participants and activities. Similarly, the lack of available space for larger gatherings of students at popular teaching times made plenary events difficult to arrange. Mix projects adapted by holding common events later in the day, integrating events into class projects, and connecting students through electronic means.

\section{Funding}

The financial support provided by the TLEF program was essential for the success of the UBC Mix project. Funds from TLEF provided the salary for the Mix Coordinator and seed funding for Mixes. The ability of Mix to provide money for small expenses such as food, transportation, and guest speaker honoraria was an important part of the success of many Mix projects.

\section{Expanding the Mix Community}

The Mix project initially focused exclusively on course instructors. As the project developed, it became evident that units without their own teaching programs or courses were interested in Mix as a mechanism to enhance their engagement with students. In particular, representatives of the UBC Sustainability Initiative and the Community Learning Initiative became active participants in UBC Mix.

\section{Sustaining the Mix Community}

A final challenge was sustaining UBC Mix beyond the funding provided by the UBC TLEF grant. To this end, UBC Mix established a partnership with UBC's Centre for Teaching, Learning and Technology (CTLT), and the UBC Mix Coordinator worked in the CTLT office to take advantage of its networking and administrative capacities. The governance of the project then transitioned into the CTLT within its community of practice framework, becoming the core foundation of the new Interdisciplinary Community of Practice (ICoP). The ICoP now meets regularly for networking events, facilitated workshops, and information-sharing sessions.

\section{Conclusion}

"Mixing" creates and enhances interdisciplinary teaching and learning opportunities at colleges and universities. UBC Mix offers a model for developing partnerships between instructors and courses to facilitate the creation of learning activities that bring students from different courses in 
different disciplines together in shared experiences. The Mix model has the advantage of operating within the framework of established programs and class schedules, while offering considerable flexibility in the form and shape of any given Mix project. Through Mix, the value of interdisciplinary learning can be expanded to a larger numbers of students through a very modest commitment of resources, while promoting cooperation and community among instructors interested in interdisciplinary teaching.

\section{Acknowledgements}

We would like to thank the following students for their involvement, Brendan Clyde, Geoff Costeloe, Sam Fenn, Claudia Goodine, Lydia Jones, and Jonathan Strang. We thank all of our colleagues who engaged with UBC Mix, particularly Luisa Canuto, Chad Hyson, and David Ng who helped us champion the project. We also acknowledge support from UBC students provided by the Teaching and Learning Enhancement Fund.

\section{Biographies}

Joanne Fox is a Senior Instructor in the Michael Smith Laboratories and the Department of Microbiology and Immunology in the Faculty of Science at the University of British Columbia. Joanne was co-lead for the UBC MIX project.

Natalie Baloy is a Ph.D. student in the Department of Anthropology in the Faculty of Arts at the University of British Columbia. Natalie was the UBC MIX project coordinator from 2011-2013.

Allen Sens is a Professor of Teaching in the Department of Political Science in the Faculty of Arts at the University of British Columbia. Allen was co-lead for the UBC MIX project.

\section{References}

Holley, K. A. (2009). Special issue: Understanding interdisciplinary challenges and opportunities in higher education. ASHE Higher Education Report, 35, 2, 1-131.

Epstein, M. J. (2004). Teaching a "humanistic" science: Reflections on interdisciplinary course design at the post-secondary level. Current Issues in Education, 7, 3, 1-11.

Klein, Julie Thompson. (2009). Creating Interdisciplinary Campus Cultures: A Model for Strength and Sustainability. John Wiley \& Sons.

Knotts, G., Henderson, L., Davidson, R. A., \& Swain, J. D. (2009). The Search for Authentic Practice Across the Disciplinary Divide. College Teaching, 57, 4, 188-196.

Letterman, M. R., \& Dugan, K. B. (2004). Team Teaching a Cross-Disciplinary Honors Course: Preparation and Development. College Teaching, 52, 2, 76-79.

Miller, K., \& Totten, I. (2009) Developing and implementing an interdisciplinary origins course at a state university. Journal of College Science Teaching, 38, 4, 24-29. 
Newell, W. H. (1994). Designing interdisciplinary courses. New Directions for Teaching and Learning, 58, 35-51.

Newswander, L. K., \& Borrego, M. (2009). Engagement in two interdisciplinary graduate programs. Higher Education: The International Journal of Higher Education and Educational Planning, 58, 4, 551-562.

Smith, Barbara L., \& John McCann, eds. (2001). Reinventing Ourselves: Interdisciplinary Education, Collaborative Learning, and Experimentation in Higher Education. Bolton, MA: Anker.

UBC (2012) Place and Promise: The UBC Plan, Student Learning, http://strategicplan.ubc.ca/the-plan/student-learning/ 